\title{
Does attention cause illusory line motion?
}

\author{
JOHN CHRISTIE and RAYMOND M. KLEIN \\ Dalhousie University, Halifax, Nova Scotia, Canada
}

\begin{abstract}
Illusory line motion (ILM) has been shown to occur when a line is presented with one end next to a previously stimulated location. The line appears to be drawn away from the site of stimulation. It has been suggested that this is because of the allocation of attention to the stimulated site. Using an endogenous attentional manipulation (a central arrow cue) with no differences in the display between the two ends of the line at the time of line presentation or immediately prior, no ILM was detected, though there was a small effect in the opposite direction. Those who have found endogenously induced ILM have used an endogenous cue based on a property of a location marker that indicated the cued location. Changing the method of cuing to one based on a property of a peripheral marker instead of a central arrow produced a small but significant report of ILM. The small magnitude of the effect, participant self-reports, and the absence of the effect in the purely endogenous condition, suggest that this was merely a bias. ILM is not generated by endogenous attention shifts.
\end{abstract}

When a line is presented all at once with one end near the location of a luminance increase or near any transient that can act as a peripheral cue, the line appears to be drawn away from the cued end (Miyauchi, Shimojo, \& Hikosaka, 1991; Schmidt, 2000). This nonveridical perception - which has been so strong that in human subjective experience (Christie \& Barresi, 2002) and cat visual cortex (Jancke, Chavane, Naaman, \& Grinvald, 2004) it is indistinguishable from real motion - has been referred to as illusory line motion (ILM). ${ }^{1}$ There have been numerous studies of the illusion that extend the original observation and seek to understand its source and nature (Downing \& Treisman, 1997; Schmidt, 2000). One leading explanation for the illusion (Hikosaka, Miyauchi, \& Shimojo, 1993a, 1993b; Schmidt \& Klein, 1997) is based on the idea that there is a gradient of accelerated arrival times at perceptual levels of processing around a cued location and that, when a line is presented (all at once) across this gradient, the difference in arrival times across the line is interpreted by motion perception systems as a drawing of the line over time. Although there are some notable exceptions to this view (e.g., P. E. Downing \& Treisman, 1997; Tse \& Cavanagh, 1995), it does serve as a general framework for the research presented here.

This illusion of motion has garnered considerable interest as a putative reflection of the locus of visual attention (Hikosaka et al., 1993b; Steinman, Steinman, \& Lehmkuhle, 1995). If it is, then, with appropriate methodology, scientists interested in assessing the locus of attention

This research was funded by a grant to R.M.K. from the National Science and Research Council of Canada. The authors thank all of the participants who volunteered their time. Experiments 1-3 were originally presented as a poster (Klein \& Christie, 1996). Correspondence should be addressed to either author at Department of Psychology, Dalhousie University, Halifax, NS, B3H 4JI Canada (e-mail: jc@or.psychology.dal .ca or ray.klein@dal.ca). would have at their disposal a very efficient tool for making such assessments. In addition, the strength of an attentional shift might be reflected in the strength of the illusion. The major focus of this study is to determine whether ILM is a reflection of the locus of attention. Although our findings should provide valuable data for understanding the source and nature of the illusion, it is not our purpose here to address these issues.

It has further been asserted that the orienting of attentional mechanisms toward the location of the luminance increase is the cause of the perceived motion within the line (Hikosaka et al., 1993b; von Grünau, Racette, \& Kwas, 1996). Studies of temporal order judgments have shown that the orienting of attention produces an acceleration of arrival times in the vicinity of the attended locus (Schneider \& Bavelier, 2003; Shore, Spence, \& Klein, 2001; Stelmach \& Herdman, 1991). Moreover, it has been well established that there is a gradient of improved performance (in response time and/or quality of information) around an attended location (C. J. Downing, 1988; McCormick \& Klein, 1990). If attention was characterized by a gradient of arrival times and, as suggested above, the illusion was mediated by such a gradient, then a line presented radiating from an attended location would be perceived as being drawn away from that site.

The following experiments test the attention hypothesis, according to which attention is a cause of ILM. Our rationale (see, also, Hecht, 1995) is situated in the fact that when attention is directed exogenously, the locus of attention and the locus of visual stimulation are confounded. Our strategy was to compare the effects upon the line motion experience from two different methods for controlling the locus of attention, called endogenous and exogenous, while obtaining independent, objective evidence on the locus of attention. Typically, ILM is induced through peripheral stimulation that is presumed to cause attention to orient toward the locus of the stimulation automatically. 
This is an exogenous cue. Endogenous cues cause the observer to strategically orient attention toward the likely location of a target in the absence of transients at that location. If ILM is generated by the allocation of attention to a locus in space, then it should be observed whether attention has been oriented to that location automatically by a peripheral flash or in a strategic manner according to instructions. On the other hand, if ILM is generated by the peripheral event per se, then it should be observed only in the exogenous cuing condition.

Although there have been studies in which ILM was observed following endogenous cuing, in some cases flaws in the implementation cast doubt on the positive findings. One such flaw is that in some studies, experienced observers (the authors) were used (Hikosaka et al., 1993b). In tests of the strength of an illusory experience as a function of a manipulation of one's own attention, it is possible that the observer's biases will influence the results. Another flaw is that eye position has not always been monitored. It has been suggested that the illusion can be induced by having one end of the line presented at fixation (Hecht, 1995). When eye position is not monitored, the findings cannot be secured against the claim that the pattern is due to the locus of fixation because gaze and attention can be dissociated (Posner, Snyder, \& Davidson, 1980 ), and observers may not know where they are fixating (Kaufman \& Richards, 1969; Shepherd, Findlay, \& Hockey, 1986). Another problem with investigations into the relationship between ILM and attention is that rarely has a fine-grained rating scale been used. When a coarse rating scale is used (e.g., one in which there are only a few categories of motion experience, such as leftward, none, rightward), participants may choose a direction merely to avoid constantly rating the drawing as "none," and these choices may be influenced by thought patterns associated with following the cue instructions.

Some studies (e.g., Hecht, 1995, Experiment 4) have manipulated attention via instruction but did not provide a direct measure that was independent of the subjective motion reports to confirm the success of the manipulation. In contrast, the present study, like Schmidt (2000), will use an independent measure to confirm the success of the attentional manipulation. Typically, attentional allocation in space is observed as improved performance in processing items at an attended location. In order to attribute ILM to accelerated arrival times in the vicinity of the attended location, performance changes due to attentional allocation should coincide with ILM.

The methods of the current study involve a dual task. From the participant's point of view, the primary task is simple detection. With endogenous control of attention, this task is used to give the participants incentive to shift attention to one of two target locations and, regardless of the mode of controlling attention performance on this task, is used to provide an independent assessment of the success of the attentional manipulation. Occasionally, instead of the detection target, a line is presented that connects the two possible target locations, and on these trials (which serve as "catch" trials for the primary task), the participant performs the secondary task of reporting on their subjective experience of motion within the line.

The terms valid and invalid are used to refer to detection targets presented at the cued location and uncued locations, respectively. If there is no directional cue, then the condition is referred to as neutral. The exogenous cue is a luminance increment in the periphery, at one of the two possible target locations, that conveys no information about the upcoming target location. In Experiments 1 through 3, the endogenous cue was an arrow presented at fixation, indicating the likely location of a detection target that was presented on most trials. In Experiment 4, the endogenous cue was based on the shape of the target location markers. The critical question is, what will be experienced on the secondary probe trials, when lines are presented occasionally in the context of the attentional manipulations described above? Will motion be perceived within these lines, and will the direction and strength of motion be affected by the locus of attention? Eye position was monitored and naive observers were tested in all four experiments reported here.

\section{EXPERIMENT 1}

In this experiment we used both exogenous and endogenous cues to orient attention and measured the effects of this orienting in time taken to detect targets presented at validly and invalidly cued locations. On probe trials, a line was presented connecting these locations. In addition to lines presented all at once (all pixels are turned on simultaneously), we also included conditions in which real line drawing occurred so that we could compare the subjective reports of ILM with a real event. ${ }^{2}$

The effect of attentional allocation was measured independently of the line motion assessment by having the participant's main task be a detection response. If participants' performance on the detection task was faster for valid than for invalid trials, it was assumed that their attention had moved to the cued location. Assuming that attention is directed roughly equally by both types of cue, the question of interest is whether the illusory motion will emanate from the cued location roughly equally using the two cuing methods. This is the finding that is predicted by the attention hypothesis.

At all times, eye position was monitored, and if a participant moved his or her eyes from fixation, the trial was terminated and not counted in the final data.

\section{Method}

Participants. Eleven university students, all with normal vision, gave informed consent and participated for a compensation of one credit point in an introductory psychology course.

Apparatus and Stimuli. A Macintosh IIx computer was used to record the data and to drive a Tektronix 603 oscilloscope upon which stimuli were displayed. The response keys and oscilloscope were all interfaced to the computer with a National Instruments NB-MIO-16h board. The data from the EyeTrac 210 eye monitor, used to assure fixation, was also collected through this board. The 


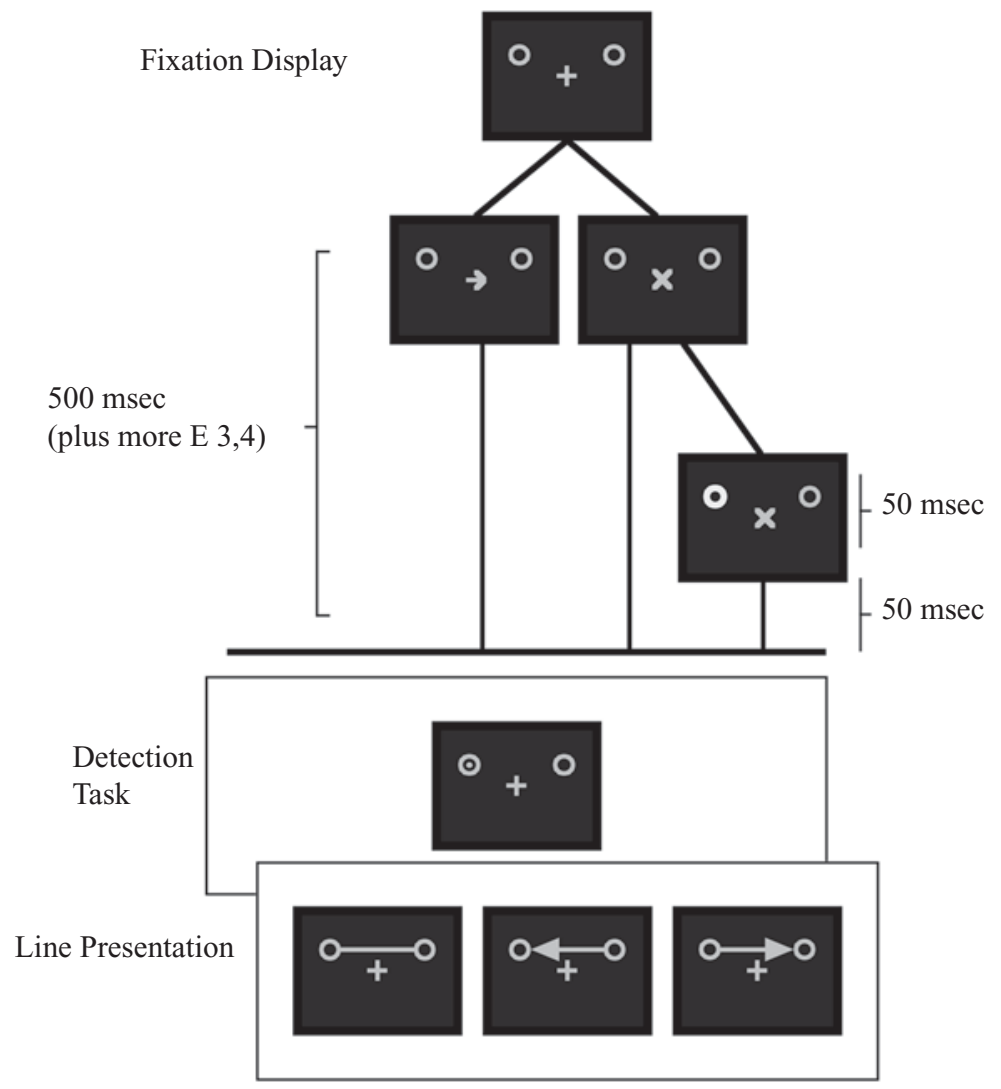

Figure 1. The layout for the sequence of events in a trial in Experiments 1 and 2. In Experiments 3 and 4, the 50-msec flash did not occur. In Experiment 4, the arrows did not occur, and one of the location markers was a square instead of a circle. The line drawing may be presented all at once or drawn from one side to the other. The arrows on the lines were not actually presented but represent drawing direction.

stimulus array at the start of each trial (see Figure 1) consisted of two $0.57^{\circ}$ circles centered $5^{\circ}$ apart, $1.1^{\circ}$ above a $0.57^{\circ}$ fixation cross. The fixation cross could turn into a left arrow, a right arrow, or an "X." These represented the endogenous left, right, and neutral cues, respectively, and were all of equal luminance. The exogenous cues consisted of a 50-msec brightening of one of the circles surrounding a target location. This brightening consisted of doubling the number of dots that composed the circle. In the center of either circle, a dot could appear for simple detection tasks. On catch trials, a line would appear connecting the two circles. It either came on all at once or was drawn from one circle to the other at a rate of $126.6 \% \mathrm{sec}$. It remained on for $1 \mathrm{sec}$. The refresh rate of the vector display was set to $250 \mathrm{~Hz}$.

After lines were presented, an assessment scale was presented, as shown in Figure 2. It consisted of a horizontal line 8.6 long with small markers at each end and a triangle positioned below the center. The participants rated the direction of line movement and strength of experience by moving the marker with buttons under their index and middle fingers for left or right, respectively. If the participant experienced no motion, they did not have to move the marker and left it at the center. If the participant experienced motion, they would move the marker to the left for leftward motion and to the right for rightward motion. To indicate stronger motion experience (slower "drawing speed"), the cursor was moved further from center. ${ }^{3}$ Once the marker was at the desired location, a button under the ring finger was used to indicate that the participant had completed the assess- ment and to end the trial. Marker positions were coded -111 for extreme left and +111 for extreme right, with the center coded as 0 and in-between values computed by linear interpolation.

Design and Procedure. There were 20 possible conditions. Five of these were to detect dot conditions that made up most (240) of the 330 trials. There were 48 detect dot trials with neutral cues. There were 72 detect dot trials with valid endogenous cues and 24 with invalid endogenous cues. There were 48 each of exogenous invalid cue and exogenous valid cue detect dot conditions. Left and right targets were used with equal frequency on these trials. The rest of the conditions, in which the dot was replaced by the line connecting the two peripheral markers, served as catch trials for the detection task. There were 15 line assessment conditions with three kinds of line (drawn leftward, drawn rightward, simultaneous) $\times$ five kinds of cue (left arrow, left flash, right arrow, right flash, neutral). There were six trials in each of these conditions.

Before the participants were run, they were required to don the eye monitors and complete a calibration procedure. They were instructed to maintain fixation on every trial and informed that if they moved their eyes before target or line presentation, the trial would be recycled and run later. If participants moved their eyes after presentation of the dot and before response execution, or after presentation of the line and before its removal, then the trial was not recycled but was rejected to ensure that the intended event probability manipulation was maintained. A movement was classified as a deviation greater than $1^{\circ}$ from fixation using a $15-\mathrm{msec}$ sampling rate of the 


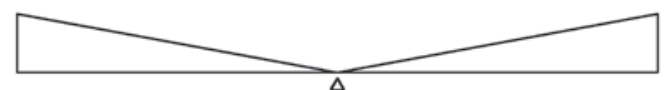

Figure 2. The motion rating screen. The participant could move the small triangle at the bottom of the figure from left to right to indicate the strength of perceived motion.

eye position. The participants were also informed about the nature of the cuing manipulation. They were told that occasional flashes might occur in the periphery, but that these were not predictive of where the target would appear. In addition, they were instructed that central arrow cues were predictive of the location of the target $75 \%$ of the time that a target appeared and that it was in their best interest to attend to the location indicated by the arrow.

Each trial was initiated with the depression and release of a foot pedal. The sequence of events that subsequently occurred are laid out in Figure 1. The participant would receive an endogenous cue that either indicated a target was likely to be presented in the left circle (left arrow) or the right circle (right arrow), or that conveyed no information (an X). Following an X (neutral) cue, there may have been a flash (exogenous cue) of one of the peripheral circles for $50 \mathrm{msec}$ with a $100-\mathrm{msec}$ flash to target (or line) SOA. The arrow cues had a $75 \%$ probability of indicating the actual target location, whereas on trials in which there was a neutral cue, with or without peripheral flashes, the target was equally likely to occur in either location. Five hundred msec after the endogenous cue, there was either a target in the center of one of the circles or a line between the circles. If the target appeared, the participants were required to press a button under their thumb as quickly as possible in order to indicate detection. If the line appeared, the participants were required to withhold responding. The line may have appeared all at once, or drawn to the left, or drawn to the right. It remained on for $500 \mathrm{msec}$. After the line was presented, it was erased, and $1 \mathrm{sec}$ later, the rating display in Figure 2 appeared, allowing the participant to indicate whether they experienced a line drawn or coming on all at once. Feedback followed each detection trial that contained the participants' response times (RT) if they made an appropriate response or one of the following error messages: "EYES MOVED" immediately following an eye movement; "WAIT FOR TARGET" immediately following an anticipation response; "USE CORRECT BUTTON" immediately following an error in which another button on the response pad was accidentally pressed; or "RESPOND SOONER" in the event that there was a missed stimulus. If they responded during the line presentation that was considered a false alarm, they immediately received the feedback "DO NOT RESPOND TO LINES." The feedback remained on the screen for $1 \mathrm{sec}$ and then the markers reappeared.

\section{Results}

No participants exceeded the criterion of $20 \%$ eye errors (in nonrecyclable trials), and the maximum amount any participant made was $10 \%$. In addition, no participants exceeded the maximum allowable average $30 \%$ false alarm rate.

Detection performance. The performance in the detection component of the experiment is presented in Figure 3. Endogenous and exogenous cuing effects were analyzed separately, with the neutral condition playing this role in each analysis. There was a main effect of the endogenous cuing manipulation on detection performance $\left[F(2,20)=10.88, M S_{\mathrm{e}}=281, p<.01\right]$. Planned comparisons revealed that the valid RT was faster than the neutral (benefit) $\left[F(1,10)=7.56, M S_{\mathrm{e}}=265, p=\right.$ $.02]$, whereas the difference between neutral RT and invalid (cost) was marginal $\left[F(1,10)=3.78, M S_{\mathrm{e}}=290\right.$,

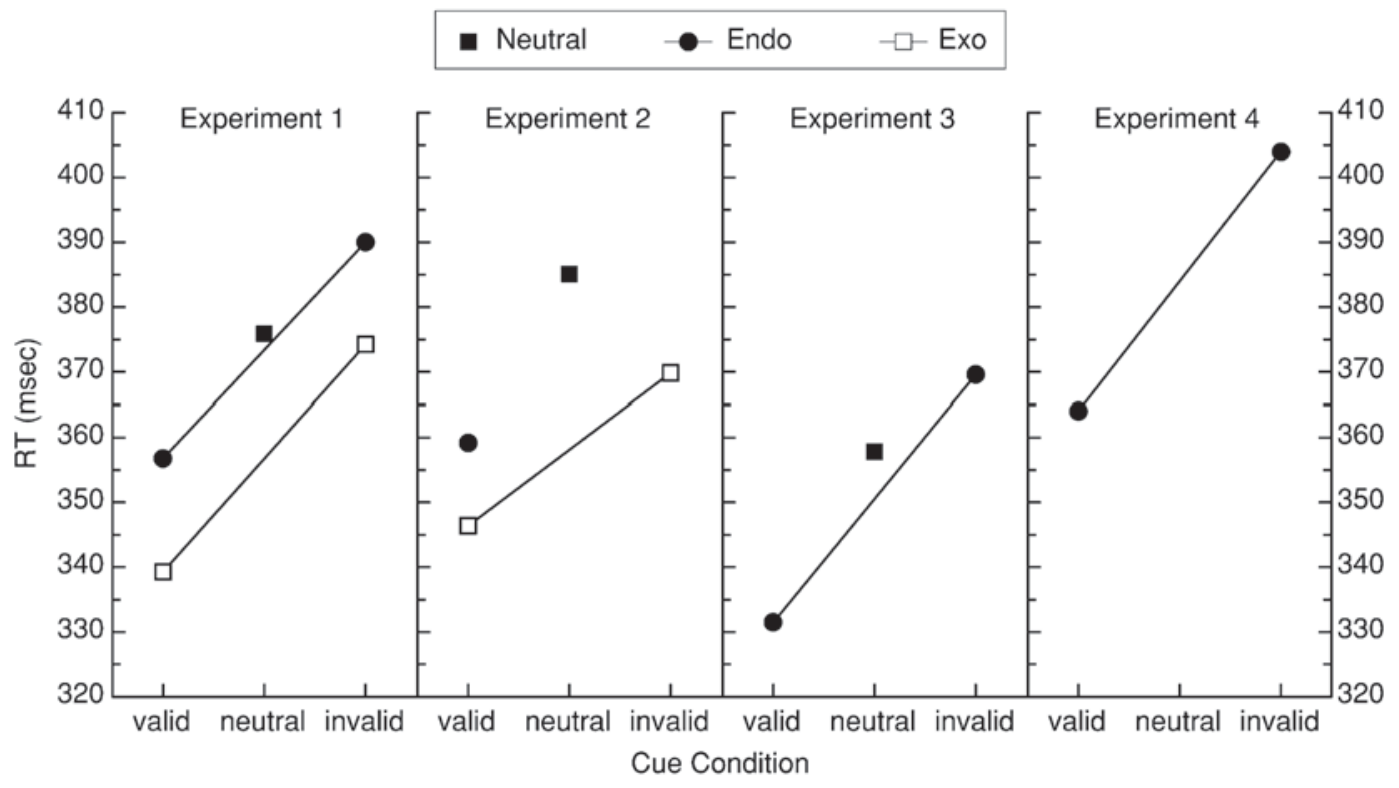

Figure 3. Detection reaction times for all experiments. Valid refers to trials in which the direction or location of the cue corresponds with that of the target; invalid refers to trials when they do not correspond. Endogenous means that the cues conveyed information to the observer about which location was likely to contain the target. Exogenous means that the cue was an uninformative flash at the cued location. 
$p=.08]$. There was also a main effect of exogenous cuing $\left[F(2,20)=36.89, M S_{\mathrm{e}}=127, p<.01\right]$. Here the benefits were significant $[F(1,10)=112.67, p<.01]$, whereas costs were not $(F<1)$.

Ignoring the neutral condition, one can test for an interaction between endogenous and exogenous cuing. There was a main effect of the type of cuing $[F(1,10)=5.6$, $\left.M S_{\mathrm{e}}=548, p=.04\right]$, with exogenous cuing causing overall faster responding than endogenous cuing. This latter effect is likely due to the flash's making participants more alert. There was no interaction between endogenous and exogenous cuing $(F<1)$. The differences between valid and invalid for the endogenous and exogenous conditions was nearly equal at 33 and $35 \mathrm{msec}$, respectively $(F<1)$.

Line assessment. The participants' ratings of line motion are shown in Figure 4. The analysis of variance (ANOVA) on which the confidence intervals are based is $\left[F(14,140)=26.8, M S_{\mathrm{e}}=592, p<.01,95 \% \mathrm{CI}=\right.$ 14.5]. As this is a novel way of showing these data, we will walk the reader through it. Focus first on the data from lines that appeared all at once (empty circles) following a neutral cue (middle of the three horizontal panels). Under these conditions, and as expected, there is no perception of motion. Staying within this panel, we can expand our view to include data from the lines that were actually drawn across the screen. These lines, too, were perceived veridically, with leftward drawn lines (triangle with vertices pointing leftward) perceived as drawn to the left and rightward drawn lines perceived as drawn to the right. The critical data from this experiment came from lines that were drawn all at once (open circles) when the participant was attending to the left or right following an exogenous (top panel) or endogenous (bottom panel) cue. Following exogenous orienting, there was an illusory line motion experience that was as strong as the real line motion experienced in the neutral condition. In addition, for lines that were actually drawn toward the cued location, the combination of illusory motion and real motion in opposite directions resulted in a net experience of no motion. For real motion in the same direction as the illusion, there was a significant increase in the strength of the motion perception. Endogenous cues had no effect on judgments of motion - these were nearly identical to what was seen in the neutral cue condition.

\section{Discussion}

The endogenous and exogenous cuing manipulations worked as predicted on the detection performance, with valid RT faster than invalid. From this, we infer that attention was directed toward the cued location and away from the uncued location. Moreover, the magnitude of the cuing effect (invalid minus valid) was approximately the same following the two kinds of cues. This provides prima facie evidence that attention was oriented toward the cued location to roughly equal degrees following endogenous and exogenous cues. Nevertheless, the perception of line motion varied dramatically as a function of cue type. With exogenous orienting, ILM was found, as predicted. The participants rated illusory line motion as strongly as real line motion and away from the attended location. In contrast, endogenously shifted attention had no effect on perceived line motion, and the small numerical difference

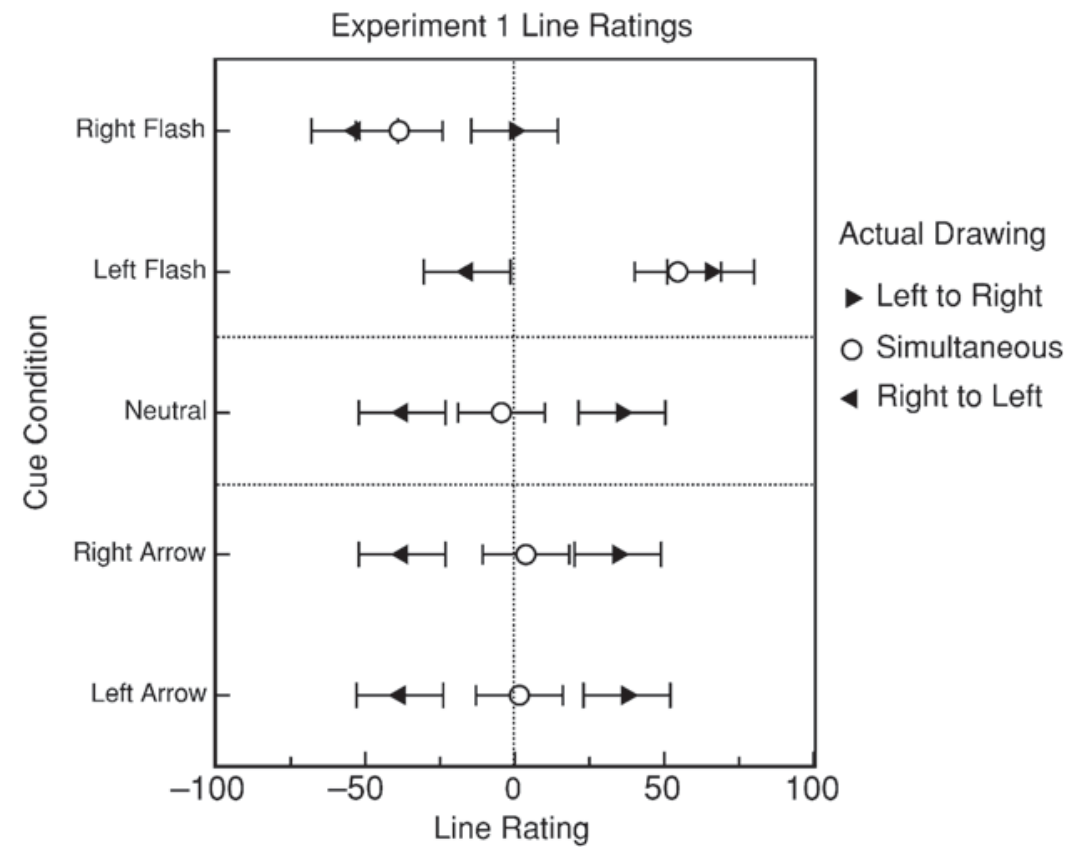

Figure 4. Line ratings for Experiment 1. The error bars represent $95 \%$ confidence intervals derived from an overall one-way ANOVA $\left[F(14,140)=26.8, M S_{\mathrm{e}}=592, p<\right.$ 0.01] (Loftus \& Masson, 1994). 
was in the direction counter to ILM. Therefore, it was tentatively concluded that it was not attention in general, but some other property of the peripheral cue, or some form of attention specific to the peripheral cues, that caused the experience of ILM.

\section{EXPERIMENT 2}

In spite of the measured equivalence of the effects of endogenous and exogenous cuing upon target detection, it might be suggested that endogenous cuing would have elicited ILM if our manipulation had been stronger. This question was tested in the next experiment by using an endogenous cue that was $100 \%$ valid.

\section{Method}

The methods were identical to those of Experiment 1 with the following exceptions. Invalid endogenously cued trials were removed, and participants were fully informed that, following an arrow cue, all targets (when presented) would be presented at the location indicated by the cue. The trials remaining were identical in number to E1. Hence there were 216 detection and 90 line judgment trials. Furthermore, after six participants were run, the remaining 11 received a very slightly different procedure. The error feedback on false alarm trials stayed on the screen for $5 \mathrm{sec}$ instead of $1 \mathrm{sec}$. This was introduced to reduce false alarm rates, which were high in the first 6 participants $(0.24$ as opposed to 0.09 in the remaining 11). One participant was dropped because the participant exceeded the $30 \%$ false alarm rate cut off.

\section{Results}

Detection performance. The pattern of performance in detection conditions was nearly identical to the corresponding conditions in Experiment 1. The results are shown in Figure 3. The endogenous cuing effect was sig- nificant, with valid faster than neutral $[F(1,15)=18.9$, $\left.M S_{\mathrm{e}}=285, p<.01\right]$. The exogenous cuing effect was also significant $\left[F(2,30)=18.6, M S_{\mathrm{e}}=327, p<.01\right]$, and, as in Experiment 1, there were no costs relative to the neutral; only benefits were significant.

Line assessment. As can be seen from the line assessment data presented in Figure 5, the line motion experience in this experiment was nearly identical to that in Experiment 1. The analysis was $[F(14,210)=34.09$, $\left.M S_{\mathrm{e}}=1043, p<.01\right]$, and the $95 \%$ confidence interval was 15.9.

\section{Discussion}

Following a $100 \%$ valid endogenous cue, which produced significant benefits, ILM was not induced, thus reinforcing the findings and conclusions of Experiment 1. A cross experiment assessment of the line ratings yielded no main effects or interactions (all $F_{\mathrm{S}}<1$ ).

\section{EXPERIMENTS 3A-3C}

The findings from Experiment 1 and Experiment 2 are rather definitive in showing that ILM is strongly experienced following exogenous cuing and apparently not experienced at all following endogenous cuing. The absence of any illusory drawing experience following endogenous cues that affect detection performance does, on the face of it, suggest that such shifts of attention are not generating any ILM. However, perhaps a weak ILM experience is generated by endogenous cues but the real line drawing and strong ILM generated from the peripheral cues produced such strong motion experiences that any endogenously generated ILM was, by comparison, overwhelmed.

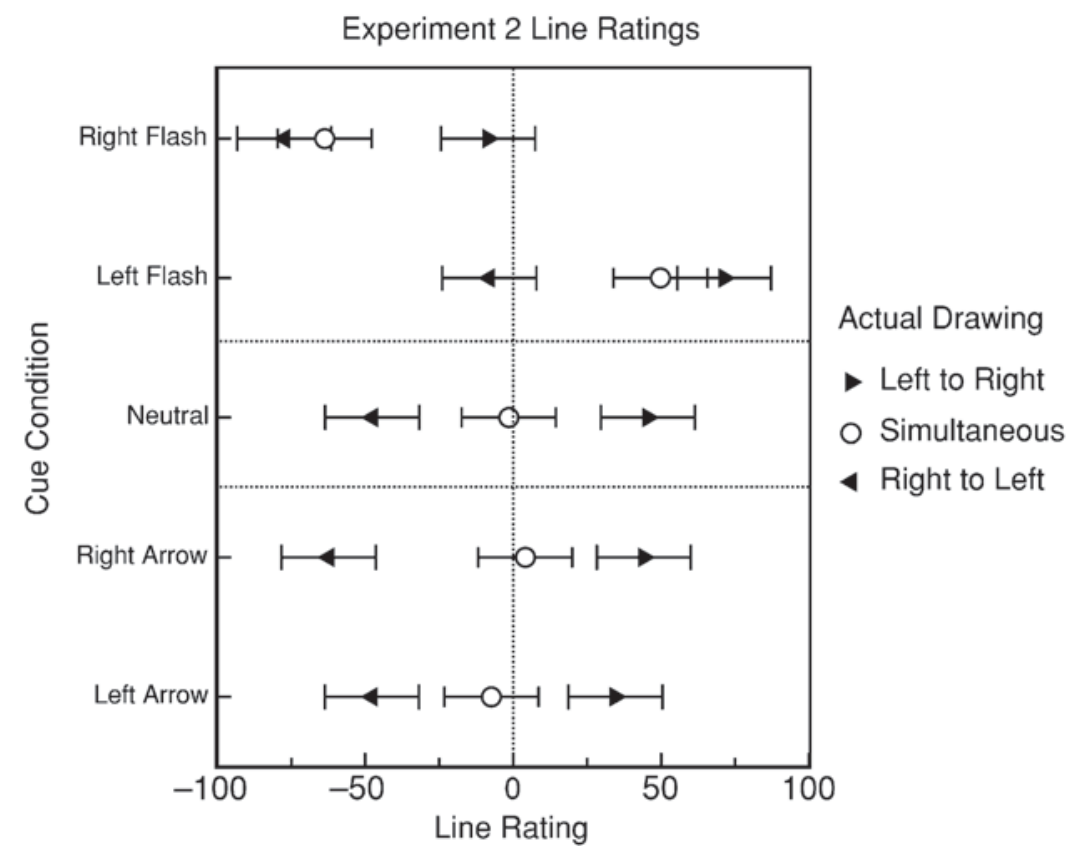

Figure 5. The line ratings in Experiment 2. 
Indeed, some previous studies that reported ILM induced by endogenous orienting also reported effects smaller than those found with exogenous orienting (P. E. Downing \& Treisman, 1997; Schmidt, 2000). We tested this "adaptation level" possibility in Experiment 3 by never presenting a strong line motion perception within the context of the experiment.

In this experiment, only static lines were presented; therefore, any experience of line motion would necessarily be illusory. This was done to insure that any small effects of illusory motion would not be washed out by the large effect of the real motion. In addition, there were no exogenous cuing conditions for the same reason.

In Experiment 3 we also added the feature that participants could wait until they felt that they had shifted their attention to the cued location before initiating stimulus presentation (cf. McCormick \& Klein, 1990; Schmidt, 2000).

\section{Method \\ Participants. A total of 44 university students participated in Experiments 3A-3C. \\ Procedure. The methods of Experiment $3 \mathrm{~A}$ were identical to those of Experiment 1 with the following exceptions. In Experi- ment 1 , the endogenous cue appeared and the 500-msec timer began upon pressing the foot pedal. In this experiment, participants initi- ated these events by pressing and then releasing the foot pedal. The participants were trained in this procedure and asked to release the foot pedal when they felt that their attention was at the location indi- cated by the arrow cue. In addition, all trials in which line drawing or exogenous cues had appeared in Experiment 1 were removed. The remaining ILM rating trials were doubled in number. Hence there were 144 detection and 36 line judgment trials. \\ Experiment $3 \mathrm{~B}$ was the same as $3 \mathrm{~A}$ but without eye monitoring. Experiment $3 \mathrm{C}$ was the same as $3 \mathrm{~B}$ but participants received the error feedback on false alarm trials for $5 \mathrm{sec}$ instead of $1 \mathrm{sec}$.}

\section{Results}

Four of the 16 participants in Experiment $3 \mathrm{~A}$ were dropped because they exceeded the maximum false alarm criterion. In Experiment 3B, 5 of the 20 were dropped for the same reason. In Experiment $3 \mathrm{C}$ - the one with the incentive not to make false alarms - all 8 participants were retained. Thus, 35 participants are included in this analysis.

There were no cross-experiment main effects or interactions (all $F_{\mathrm{S}}<1$ ). Therefore, the following analyses were conducted and reported as if there had been just one big experiment.

Detection performance. The endogenous cuing manipulation again affected detection performance in the predicted fashion $\left[F(2,68)=17.1, M S_{\mathrm{e}}=780, p<.01\right]$. These results can be viewed in Figure 3, where it can be seen that the pattern of performance is similar to the endogenous cuing of Experiment 1.

Line assessment. As can be seen in Figure 6, there were no effects of endogenous cuing on line motion assessment $\left[F(2,68)=0.13, M S_{\mathrm{e}}=349, p<.01\right], 95 \%$ confidence interval is 6.3. In this particular instance, however, the rating was in a direction that is the same as that predicted for ILM. A sign test, conducted to determine whether participants experienced or did not experience motion in accord with the attention hypothesis and effect, revealed that only 18 of the 35 participants reported a net motion experience consistent with the attention hypothesis $(p=.51)$.

\section{Discussion}

Again, there was no effect of endogenous orienting of attention on the perception of lines, but there was a measurable facilitation of performance in the attended area. The strong motion percepts generated in Experiment 1 and Experiment 2 were not masking a small illusion in the endogenous cuing conditions. There simply was none to be found.

The motion ratings of simultaneously presented lines following endogenous cues in Experiments 1, 2, and 3 were calculated by subtracting right cue from left cue judgments. This measure represents the overall tendency for simultaneous lines to be experienced as drawn in a consistent direction from the cue (with + representing "away from the cued location"). In contrast to the predictions of the attention hypothesis, the mean ratings indicated a weak tendency to perceive lines drawn toward the endogenously cued location $(-2)$, suggesting that the lack of an effect is not a power issue.

Although we feel it is an unlikely possibility, our use of a continuous scale, and the consequent increase in measurement variability, could somehow have obscured an endogenous cuing effect on line motion perception. Partly to test this possibility, and partly to enhance comparability with previous studies that used a much coarser rating scale, the nearly "continuous" ratings (from -111 to +111 ) for each judgment were transformed as follows: A value of 1.0 was assigned to all rightward judgments, regardless of magnitude; a value of -1.0 was assigned to

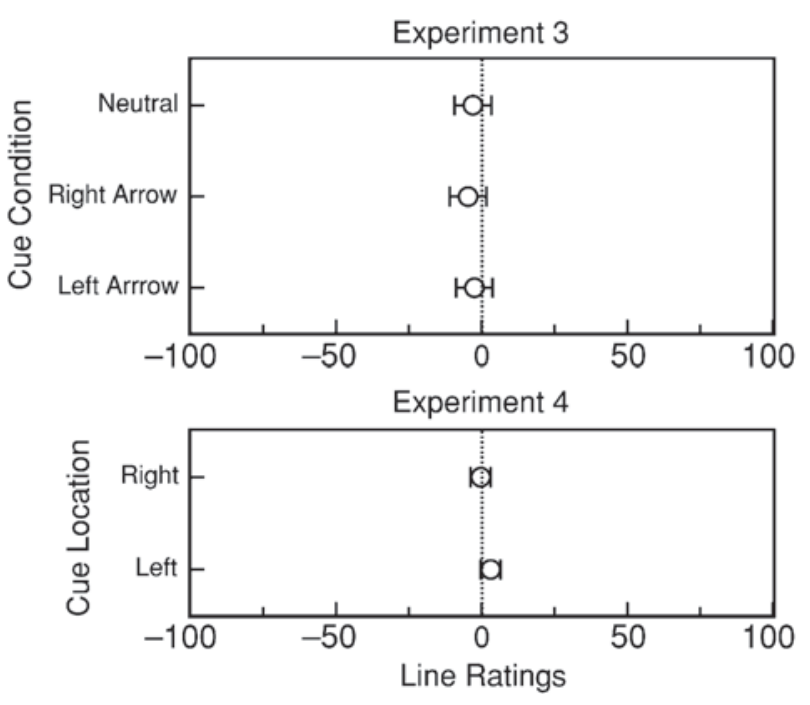

Figure 6. The line ratings from Experiments 3 and 4. In Experiment 4 , the rating of lines precued to the left marker was more rightward than ratings of lines precued to the right marker. 
all leftward judgments; and values of zero (no directional motion experience) remained the same. Means by participant of these transformed scores were then analyzed across all participants for consistency using a sign test. Using this measure, 38 of 62 participants reported motion toward the attended location, $p=.09$, which is a trend in the direction opposite from that predicted by the attention hypothesis. When exogenous cuing was subjected to the same analysis, $100 \%$ of the participants reported line motion away from the cued location.

This series of demonstrations of no effect of endogenous attention upon line motion perception is in stark contrast to some previous studies (P. E. Downing \& Treisman, 1997; Hikosaka et al., 1993b; Schmidt, 2000). One methodologically strong example of a finding of ILM with endogenous orienting is Schmidt (2000). He monitored eye movements, had participants respond to targets in cued and marked locations, and followed the general methodological improvements proposed here for studying ILM and endogenous cuing. Our evaluation of the literature leads us to suggest that, in contrast to these studies, the reason we do not find support for the attention hypothesis may reside in the endogenous cuing method used. In Experiments $1-3$, our participants were endogenously cued to attend a location by means of an informative arrow presented at fixation. As far as we know, all studies exploring the effect of endogenous orienting upon ILM, prior to ours, had used featural differences between two peripheral markers combined with a feature-driven attentional control setting (P. E. Downing \& Treisman, 1997; Hikosaka et al., 1993b; Schmidt, 2000). Experiment 4 was designed to see if, when we used this methodology, we, too, could obtain evidence for the attention hypothesis.

\section{EXPERIMENT 4}

It has been surmised that the finding of endogenously induced ILM may depend on there being a featural difference between the location markers where targets might appear in combination with the use of this difference to guide attention. This suggestion leads to an interesting alternative explanation for endogenously induced ILM, one that builds on Farah's (1990) suggestion that there may be separate object- and space-based attention systems. Attention may be distributed differently when it is applied to objects rather than locations in space (Egly, Driver, \& Rafal, 1994; Vecera \& Farah, 1994). In particular, such object-directed attention may be more akin to that rallied by a peripheral stimulus. We are aware of one study that supports this suggestion. Lambert and Duddy (2002) implemented endogenous cuing, as had been done in the prior attention and ILM literature, wherein two different peripheral stimuli were presented and the participant was informed that one stimulus was likely to contain the target. They found that this method of manipulating attention endogenously differed qualitatively from that using central arrow cues, including causing very fast orienting (they were effective at 0 -msec SOAs).
The present study is very similar to Experiment 3A. However, unlike all of the previous experiments, the endogenous cue was not a central arrow appearing at fixation but rather was contained in the visual differences between the two location markers on the screen. In Experiments 1 through 3, there were two circle markers on the screen, indicating the potential target locations. In this experiment, one marker was a circle and the other was a square. For half of the participants, the likely location for the detection target was in the square, whereas for the other half, it was in the circle. The participants were instructed to attend the square or circle, respectively, while maintaining gaze on the fixation stimulus.

\section{Method}

Participants. Twenty-five individuals volunteered for the experiment. After the exclusion of the six participants whose overall false alarm rates were above the criterion, the data of 19 participants were available for analysis.

Apparatus. The apparatus was the same as in Experiment 3 except that on each trial one of the markers on the screen was randomly selected to be a square. The square had the same perimeter as the circle.

Procedure. The procedure differed from Experiment 3 as follows: The rest period between trials that contained the fixation display with both markers also inherently contained the endogenous cue. Therefore, trial initiation to target onset interval was reduced to the same fixed $500 \mathrm{msec}$ after the button press used in Experiments 1 and 2. After trial initiation, the central fixation cross always turned into the " $X$ " used as a neutral cue in Experiments 1-3. There were no neutral cue trials because the markers were always different from one another. There were 128 dot detection trials, of which 96 were valid and the remaining 32 trials were line drawing trials. Finally, participants were randomly assigned to attend square or to attend circle conditions. Eye position was monitored as in Experiments 1,2 , and $3 \mathrm{~A}$.

\section{Results}

As can be seen in Figure 3, there were significant cuing effects with valid faster than invalid targets $[F(1,18)=$ $\left.5.6, M S_{\mathrm{e}}=2635, p=.03\right]$, just as in the previous experiments using arrow cues.

The line motion ratings, in Figure 6, are numerically in the direction predicted by the attention hypothesis but not significant in an ANOVA using the magnitude scores $\left[F(1,18)=2.24, M S_{\mathrm{e}}=49.2, p=.15\right]$. However, when a sign test was conducted to test whether participants experienced motion in the direction predicted by the attention hypothesis, the effect was significant: 15 of 19 successes $(p=.02)$. When the trial-by-trial ratings were coded coarsely as described previously, the ANOVA was significant $\left[F(1,18)=7.04, M S_{\mathrm{e}}=0.027, p=.02\right]$, and the net motion experience was consistent with the attention hypothesis. As with the sign test based on the original ratings, the sign test based on coarse coding of the ratings was also significant (16 of 19).

\section{Discussion}

We have been able to replicate previous findings of ILM with endogenous cues. To generate the effect, it appears necessary to implement the endogenous cuing by a 
differentiation between items or stimuli in the periphery. Although the effect was very small and not significant when the full rating scale was preserved, it was, nevertheless, fairly consistently obtained when the magnitudes were ignored on a participant or trial basis.

We would like to be able to assert that we have found that attentional cuing has different properties when it is based on differentiated objects instead of location. This would imply that object-based cuing speeds signals at the cued location whereas location-based cuing does not, it is also possible that our finding merely reflects a bias linked to the method of implementing the endogenous cuing. This explanation will be explored in the general discussion.

\section{GENERAL DISCUSSION}

It was found that the exogenous cues we used to orient attention produced strong percepts of ILM that were roughly equivalent in magnitude to actually drawn lines (velocity of $126.6^{\circ} / \mathrm{sec}$ ). There was no evidence to support the view that endogenous orienting of attention causes a change in the way lines are perceived. Based on these findings, we cannot accept the general hypothesis that attention causes ILM. The ILM we observed in the exogenous orienting conditions must be due to stimulus properties of the cue or to special properties unique to exogenous attention mechanisms not shared with endogenous mechanisms (Briand \& Klein, 1987; Müller \& Rabbitt, 1989; for a review, see Klein, 2004). In Experiment 4, wherein the endogenous cuing was based on differentiating peripheral objects, ILM ratings, though weak in magnitude, were consistent with the attention hypothesis. This might lead one to conclude that the hypothesis is correct when attention is allocated in this manner. Before we explain why we disagree with this conclusion, we will briefly address similar findings in the literature.

Comparing to other experiments is difficult for various reasons. Hikosaka et al. (1993b) used a simple toward or away rating. This two-alternative, forced-choice method provides no way to estimate the magnitude of the effect. One might try to compare this kind of rating to the sign test analysis of Experiment 4. It was found that 16 of 19 participants reported line motion in a direction consistent with ILM. Those who have used scales with more categories than did Hikosaka et al. (1993b) have also reported small amounts of ILM. Schmidt (2000, Experiment 1) used a 1- to 9-point scale for rating the strength of the illusion with endogenous cues and found ratings between 1 and 2 (closer to 1). P. E. Downing and Treisman (1997) also obtained small magnitudes for the ratings of endogenously induced ILM on a 9-point scale $(<1)$. Schmidt's (2000) Experiments 2-5, performed experiments with a continuous $( \pm 100$ point) scale, just as we did. The largest magnitude effect he found from endogenous cuing was approximately 18 . In that study, however, experienced observers were among those who took part, and such observers may rate the motion more strongly than do naive observers (this was not analyzed). His ratings for exogenous cue conditions were much higher, 37 . Another factor potentially contributing to Schmidt's getting larger rating values with endogenous cues than we did was that Schmidt encouraged participants to use the entire scale, whereas we did not.

Figure 7 shows the distribution of the perceptual experiences (line motion ratings) from selected conditions in all of the experiments. In the top two panels, one can see that the perceptual experiences produced by exogenous cues and actual drawing are indistinguishable. This is not surprising, given that past direct subjective reports have indicated that real and illusory motion are difficult to differentiate (Christie \& Barresi, 2002) and that they activate similar neural mechanisms (Jancke et al., 2004). This is further reinforced by the numerical magnitude of the effects' being so similar for real and exogenous cues.

The attention hypothesis, though, is refuted by the data from the endogenous conditions. The perceptual experiences from endogenous arrow cues is highly similar to that following neutral cues. Even though reaction times on the detection task were strongly influenced by the endogenous arrows - indeed as strongly as by the exogenous cues - it is very clear from the data presented thus far, and magnified in Figure 7, that there is little to no effect of the arrow cues on line ratings. When attention is endogenously directed on the basis of object properties (Experiment 4), as illustrated in the bottom panel, one can see the slight deviation in the distribution of ratings that leads to the statistically significant effect. Comparison of this pattern with that obtained from the real motion and that elicited by exogenous cues, however, reveals what we believe must be an entirely different quality of perceptual experience.

Figure 7 clearly indicates that if there is an illusion with endogenous cues, it is extremely small and variable. Consider the possibility that there is no endogenously induced illusion at all. It is possible that the rating consistency is a by-product of bias introduced by the cuing manipulation combined with the absence of any strong motion experience. In fact, we believe such a bias to be the most likely potential explanation for those effects. When participants see an arrow indicating the side where the target is likely to be presented, this induces a directional pattern. Participants think "left" when using a left arrow cue. When rating an ambiguous presentation of a line, participants may tend to rate it in the direction of the arrow. This was the trend of line ratings in Experiments 1 through 3. Conversely, when participants are attending an object, they may tend to think of that as the source of activity. If an ambiguous line is presented, and a direction is estimated, then it may be thought to start at the source, or cued end.

Nevertheless, an effect that has been called ILM from endogenous orienting in previous studies has been found, and it cannot be ruled out that there is a very weak ILM experience in the present Experiment 4. It is possible, furthermore, that this is caused by components of endogenous orienting specific to cases where items can be parsed as separate objects, but the amount of illusion is 


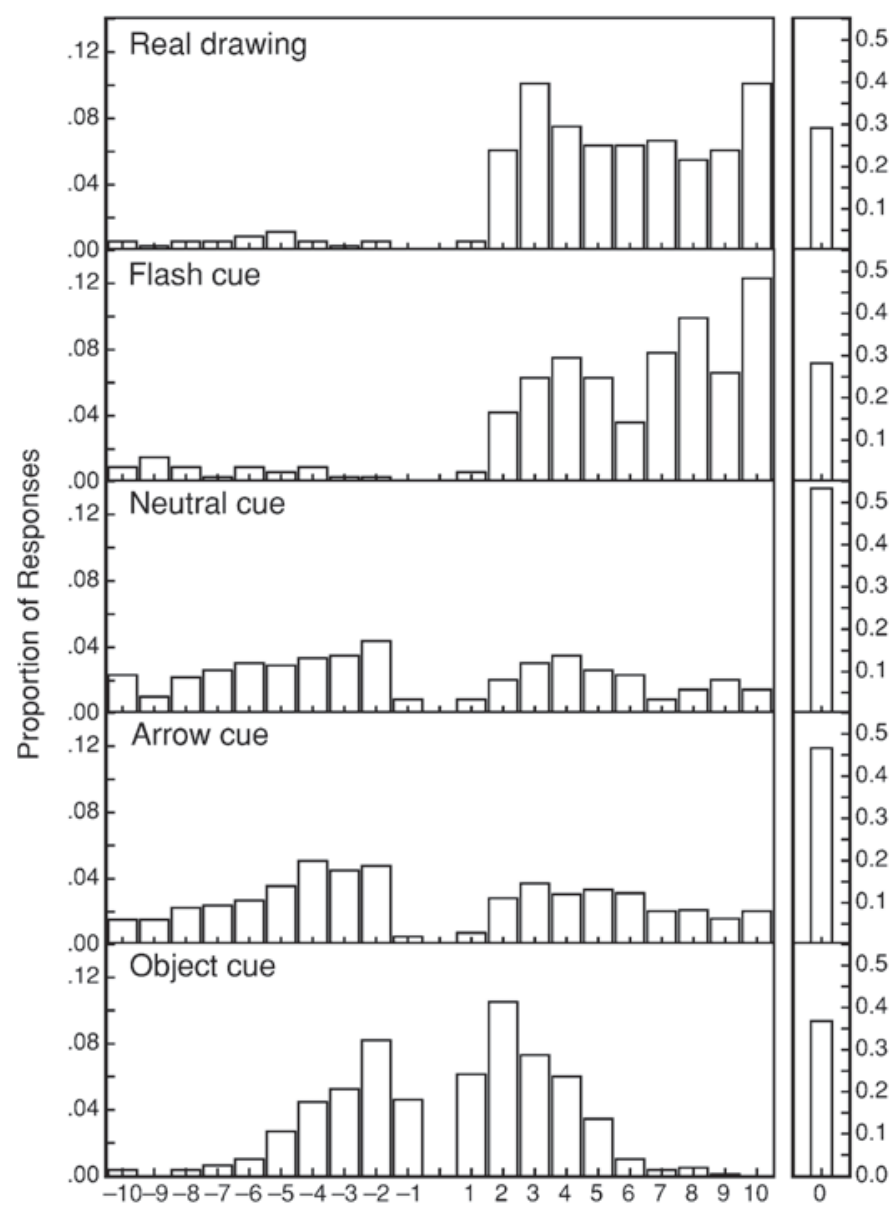

Figure 7. Distributions of line motion ratings elicited by selected conditions from Experiments 1-4. In the panels on the left, negative and positive ratings have been sorted into deciles. Panels on the right display the proportions of zero ratings. The top panel displays ratings from lines that were actually drawn following neutral cues in Experiments 1 and 2. Here, positive ratings reflect motion experiences consistent with the actual motion; negative ratings reflect erroneous percepts. The second panel displays ratings of simultaneous lines following exogenous cues in Experiments 1 and 2 . Here, positive ratings indicate motion away from the cue and negative ratings indicate motion toward the cue. The third panel displays ratings of simultaneous lines following neutral cues in Experiments 1-3. Because there was no direction (cue or real motion) in this panel, negative ratings correspond to leftward motion and positive ratings to rightward motion. The fourth panel displays ratings from simultaneous lines following endogenous arrow cues in Experiments 1-3. The bottom panel displays ratings from simultaneous lines following endogenous object cues in Experiment 4. In the latter two panels, positive ratings correspond with the attention hypothesis.

extremely small and qualitatively dissimilar from that found with exogenous cues. Because the effect of the endogenous and exogenous cues upon detection performance is the same, regardless of which cue is used, these findings definitively show that attention is not the source of ILM. When we consider that the proposal that the proximal cause of ILM is a gradient of arrival times (Schmidt \& Klein, 1997), recent findings from temporal judgment tasks provide strong support for the conclusion that this in turn is caused by local stimulation. Using sev- eral measures to minimize the contribution of response bias in a visual temporal order judgment task, Shore et al. (2001), for example, found a much greater effect upon arrival times from purely exogenous cues $(61 \mathrm{msec})$ than from an endogenous manipulation of attention $(17 \mathrm{msec})$. Schneider and Bavelier (2003), although obtaining robust arrival time effects from peripheral cues, found almost no effect of endogenous cuing in a simultaneity judgment task, which they asserted was even more immune to response bias than the methods used by Shore et al. (2001). 
There is also converging evidence that attention is not the source of ILM when exogenous cues elicit inhibition of return (IOR). In Schmidt (1996), the time course of attention shifts due to peripheral, uninformative (exogenous) cues was assessed with a manual response task. At SOAs ranging from 300 to $1,100 \mathrm{msec}$, performance was better at uncued locations (IOR). Despite this performance decrement at the cued location, ILM was found away from this location at all SOAs in this range. Hence, it is unlikely that attention is causing both ILM and IOR in those experiments. Endogenous attention systems are an unlikely candidate because the participant is presumed to be attempting not to attend the cued location. Exogenous attention is an unlikely candidate because the time between cue onset and line onset is too long for attention to still be at the cued location (as verified by Schmidt's own performance measures). We are aware of no cases in the literature in which purely exogenous orienting persists for extended periods of time. Even when exogenous and endogenous attention mechanisms are rallied into action in tandem by an informative peripheral cue, it has been demonstrated (Nakayama \& MacKeben, 1989) that effects traditionally associated with a purely exogenous shift of attention (transient) dissipate very shortly after the cue (around $200 \mathrm{msec}$ ). Indeed, the attention that remains at the cued location takes on properties more similar to those attributed to endogenous attention (Briand, 1998).

In conclusion, ILM is not induced by a spatial endogenous cue. ILM can be induced by a peripheral flash cue that also rallies attention. A weak effect that could be interpreted as ILM occurs when peripheral object-based endogenous cues are used, replicating prior literature. After reviewing the literature, we interpreted these findings to conclude that ILM is not likely caused by attention per se, either endogenously or exogenously. A strong conclusion can be made that ILM is not caused by spatial endogenous cues. A less clear picture arises when considering objectbased endogenous cues, and it may be something about object differentiation that induces the very weak ILM observed with this cuing method, or perhaps there is no ILM at all, but merely a bias induced by the nature of the cues.

\section{REFERENCES}

Briand, K. A. (1998). Feature integration and spatial attention: More evidence of a dissociation between endogenous and exogenous orienting. Journal of Experimental Psychology: Human Perception \& Performance, 24, 1243-1256.

Briand, K. A., \& Klein, R. M. (1987). Is Posner's "beam” the same as Treisman's "glue"?: On the relation between visual orienting and feature integration theory. Journal of Experimental Psychology: Human Perception \& Performance, 13, 228-241.

Christie, J., \& BARRESI, J. (2002). Using illusory line motion to differentiate misrepresentation (Stalinesque) and misremembering (Orwellian) accounts of consciousness. Consciousness \& Cognition, 11, 347-365.

DownING, C. J. (1988). Expectancy and visual-spatial attention: Effects on perceptual quality. Journal of Experimental Psychology: Human Perception \& Performance, 14, 188-202.

Downing, P. E., \& Treisman, A. M. (1997). The line-motion illusion: Attention or impletion? Journal of Experimental Psychology: Human Perception \& Performance, 23, 768-779.

Egly, R., Driver, J., \& Rafal, R. D. (1994). Shifting visual attention between objects and locations: Evidence from normal and neurologi- cal populations. Journal of Experimental Psychology: General, 123, 161-177.

FARAH, M. J. (1990). Visual agnosia: Disorders of object recognition and what they tell us about normal vision. Cambridge, MA: MIT Press.

Hecht, H. (1995). Retinal, attentional, and causal aspects of illusoryline motion directionality. Psychological Research, 57, 70-79.

Hikosaka, O., Miyauchi, S., \& Shimojo, S. (1993a). Focal visual attention produces illusory temporal order and motion sensation. Vision Research, 33, 1219-1240.

Hikosaka, O., Miyauchi, S., \& Shimojo, S. (1993b). Voluntary and stimulus-induced attention detected as motion sensation. Perception, 22, 517-526.

Jancke, D., Chavane, F., Naaman, S., \& Grinvald, A. (2004). Imaging the cortical correlates of illusion in early visual cortex. Nature, 429, 423-426.

Kaufman, L., \& Richards, W. (1969). Spontaneous fixation tendencies for visual forms. Perception \& Psychophysics, 5, 85-88.

KLeIN, R. M. (2004). On the control of visual orienting. In M. I. Posner (Ed.), Cognitive neuroscience of attention (pp. 29-44). New York: Guilford.

Klein, R. M., \& Christie, J. (1996). Comparison of illusory line motion following exogenous and endogenous orienting of attention. Investigative Ophthalmology \& Visual Science, 37, S530.

LAMBERT, A., \& DUdDY, M. (2002). Visual orienting with central and peripheral precues: Deconfounding the contributions of cue eccentricity, cue discrimination and spatial correspondence. Visual Cognition, 9, 303-336.

Loftus, G. R., \& Masson, M. E. (1994). Using confidence intervals in within-subject designs. Psychonomic Bulletin \& Review, 1, 476-490.

McCoRmick, P. A., \& KLEIN, R. A. (1990). The spatial distribution of attention during covert visual orienting. Acta Psychologica, 75, 225-242.

Miyauchi, S., Shimojo, S., \& Hikosaka, O. (1991). Attention-related motion sensation is produced by local facilitation of detection [Abstract]. Investigative Ophthalmology \& Visual Sciences, 32, 716.

MülleR, H. J., \& RabBitT, P. M. A. (1989). Reflexive and voluntary orienting of visual attention: Time course of activation and resistance to interruption. Journal of Experimental Psychology: Human Perception \& Performance, 15, 315-330.

NaKayama, K., \& MacKeben, M. (1989). Sustained and transient components of focal visual attention. Vision Research, 29, 1631-1647.

Posner, M. I., SNyder, C. R. R., \& Davidson, B. J. (1980). Attention and the detection of signals. Journal of Experimental Psychology: General, 109, 160-174.

SCHMid, W. C. (1996). Inhibition of return is not detected using illusory line motion. Perception \& Psychophysics, 58, 6, 883-898.

SCHMid, W. C. (2000). Endogenous attention and illusory line motion reexamined. Journal of Experimental Psychology: Human Perception \& Performance, 26, 980-996.

SchmidT, W. C., \& KLeIN, R. M. (1997). A spatial gradient of acceleration and temporal extension underlies three illusions of motion. Perception, 26, 857-874.

SCHNEIDER, K. A., \& BAVELIER, D. (2003). Components of visual prior entry. Cognitive Psychology, 47, 333-366.

Shepherd, M., Findlay, J. M., \& Hockey, R. J. (1986). The relationship between eye movements and spatial attention. Quarterly Journal of Experimental Psychology, 38, 475-491.

Shore, D., SPence, C., \& Klein, R. (2001). Visual prior entry. Psychological Science, 12, 205-212.

Steinman, B. A., Steinman, S. B., \& Lehmkuhle, S. (1995). Visual attention mechanisms show a center-surround organization. Vision Research, 35, 1859-1869.

Stelmach, L. B., \& Herdman, C. M. (1991). Directed attention and perception of temporal order. Journal of Experimental Psychology: Human Perception \& Performance, 17, 539-550.

Tse, P., \& Cavanagh P. (1995). Line motion occurs after surface parsing. Investigative Ophthalmology \& Visual Science, 36, S417-S417.

Vecera, S. P., \& FARAH, M. J. (1994). Does visual attention select objects or locations? Journal of Experimental Psychology: General, 123, 146-160.

von Grünau, M., Racette, L., \& Kwas, M. (1996). Measuring the attentional speed-up in the motion induction effect. Vision Research, 36, 2433-2446. 


\section{NOTES}

1.The basic illusion has also been identified as induced motion, morphing motion, shooting line, and line impletion among others.

2.In a previous study, real line motion and exogenously induced ILM were compared, and real line motion sufficient to cancel the illusion was determined. The duration of real line drawing used in the present study was selected on the basis of these previous findings.

3.Readers familiar with the range of experiences sometimes reported in ILM experiments may wonder whether our participants may have experienced two lines colliding in the center (Schmidt \& Klein, 1997) or two lines growing out from the center (Hecht, 1995). The authors and two others in our lab, who had not participated in the experiments, reported no subjective experience of either of these illusions. During debriefing, no participant spontaneously reported either of these experiences.

(Manuscript submitted May 18, 2004;

revision accepted for publication December 3, 2004.) 